\title{
High-dose Parenteral Thiamine in Treatment of Wernicke's Encephalopathy: Case Series and Review of the Literature
}

\author{
ANDREW NISHIMOTO ${ }^{1,2}$, JUSTIN USERY ${ }^{1,2}$, JOHN C. WINTON ${ }^{3}$ and JENNIFER TWILLA ${ }^{1,2}$ \\ ${ }^{1}$ Department of Pharmacy, Methodist University Hospital, Memphis, TN, U.S.A.; \\ ${ }^{2}$ Department of Clinical Pharmacy, University of Tennessee, Memphis, TN, U.S.A.; \\ ${ }^{3}$ Inpatient Physicians, Methodist Germantown Hospital, Memphis, TN, U.S.A.
}

\begin{abstract}
Background: Thiamine deficiency can lead to Wernicke's encephalopathy (WE), an acute and potentially life-threatening neurological disorder. Even though the main treatment modality for WE consists of thiamine replacement, evidence supporting an optimal dosing strategy and duration is unclear. Patients and Methods: We present a single-center case series of eleven patients that were admitted with possible WE and treated with high-dose parenteral thiamine. Results: Patients with suspected WE were treated with $\geq 500 \mathrm{mg}$ intravenous thiamine for a median of 3 days with $73 \%$ of patients (eight out of eleven) displaying symptom resolution or improvement after treatment. No significant correlation between symptom resolution and timing of highdose thiamine initiation (median=92 h) was identified. In patients whose symptoms resolved compared to those whose symptoms did not, there were no differences in patient variables nor adverse effects related to thiamine treatment. Conclusion: High-dose thiamine ( $\geq 500 \mathrm{mg}$ ) appears safe and efficacious for use in patients with suspected WE.
\end{abstract}

Wernicke's encephalopathy is an acute neuropsychiatric disorder that occurs as a result of thiamine (vitamin B1) deficiency. First characterized by Carl Wernicke in 1881, traditional signs and symptoms of the disorder include altered mental status, ataxia, and ocular signs, including nystagmus and ophthalmoplegia (1). These classical symptoms may be present in only about $16-38 \%$ of Wernicke's patients, though Wernicke's encephalopathy can

This article is freely accessible online.

Correspondence to: Dr. Jennifer Twilla, Pharm.D., BCPS. Department of Pharmacy, Methodist University Hospital, 1265 Union Avenue, Memphis, TN, 38104, U.S.A. Tel: +19015168170 , Fax: +1 9015168178, e-mail: Jennifer.Twilla@mlh.org

Key Words: Thiamine, vitamin B1, Wernicke's encephalopathy, alcohol. be further characterized by distinct patterns of alterations seen by magnetic resonance imaging (MRI) (2-4).

Wernicke's encephalopathy is associated with significant morbidity and mortality, with death reported in up to $17-20 \%$ of patients $(5,6)$. About $80-85 \%$ of patients who survive develop a chronic disorder of severe memory deficits with amnestic states that include learning defects and short term memory loss. This condition, known as Korsakoff's psychosis, often follows Wernicke's encephalopathy, which collectively is known as Wernicke-Korsakoff Syndrome (WKS).

Treatment for WKS involves administration of thiamine, even though firm consensus on the optimal dose, frequency, route and duration has not yet been established. Typical suggested regimens include high-dose thiamine ( $\geq 500 \mathrm{mg}$ ) prescribed intravenously three times a day for two to three days initially with additional treatment doses based on clinical response (7). These doses are several-times greater than the recommend daily thiamine allowance of $1.1 \mathrm{mg}$ in adult females and $1.2 \mathrm{mg}$ in adult males (8). In this case series, we retrospectively review eleven patients treated with high-dose IV thiamine for suspected Wernicke's encephalopathy to provide additional information on the management as well as clinical correlation and risk factors associated with WKS.

\section{Patients and Methods}

An electronic report searching for intravenous thiamine use in patients with ICD-9 codes for thiamine deficiency (265.1) and/or alcohol amnestic disorder (291.1) was generated. Patients admitted to Methodist University Hospital in Memphis, TN from 2010-2013 were reviewed. The report provided a list of patients from which we identified and retrospectively analyzed the electronic charts of their past admission during which high-dose thiamine was administered. Demographic information, thiamine regimen, relevant past medical history, pertinent laboratory values, hospital course, and treatment outcome was collected for each patient in this case series. Excluded from our analysis were patients that did not receive high-dose thiamine therapy or they had confounding comorbidities which obfuscated the diagnosis of WKS or resolution of symptoms. In our study, we defined high-dose thiamine therapy as $\geq 500 \mathrm{mg}$ parenteral thiamine per day. Time of treatment initiation was defined as the time from symptom 
onset until treatment with high-dose thiamine. In patients who were symptomatic prior to being admitted, time of hospital admission was used as time of symptom onset. Resolution of symptoms was interpreted based on chart documentation by the physician and included complete or partial improvement in patients' initial symptoms.

\section{Results}

Among the 32 patients which met our search criteria, 14 received high-dose parenteral thiamine ( $\geq 500 \mathrm{mg}$ thiamine/day) initially (see Table I). Wernicke's encephalopathy was suspected or diagnosed in 12 patients. One patient was excluded from our analysis due to alternative diagnoses confounding symptom resolution, leading to 11 patients included in the case series review. The median age of the patients (five males, six females) was 58 years. Thiamine levels were measured in 8 out of the 11 patients $(73 \%)$ with an appropriate measurable thiamine level detectable in only one patient $(22 \mathrm{nmol} / \mathrm{L})$. Undetectable thiamine levels were present in 5 out of the 11 patients. In two patients, intravenous thiamine had been administered before blood was drawn and the thiamine level returned abnormally high (94 and 507 $\mathrm{nmol} / \mathrm{l})$. The median baseline magnesium level was $1.8 \mathrm{mg} / \mathrm{dl}$, and seven patients had magnesium replaced during their hospitalization (median magnesium level was 1.8 in magnesium-treated patients and 1.85 in the untreated group).

In the treatment of Wernicke's encephalopathy, all patients received $500 \mathrm{mg}$ IV thiamine although the number of doses and duration of treatment varied. Six patients received the high-dose thiamine as the initial therapy for suspected Wernicke's encephalopathy. In five patients, thiamine was started prior to suspicion of Wernicke's, and of those patients, all received a standard dose of $100 \mathrm{mg}$ thiamine daily either for thiamine deficiency or prophylaxis for delirium tremens. Overall, patients were treated a median of three days with high-dose thiamine.

Upon discharge, symptoms had resolved in 7 out of 11 (63.4\%) patients. Eight (73\%) patients were discharged with thiamine, half of which did not have resolution of symptoms. Korsakoff's psychosis was noted in one patient during their admission. In this patient, whose hospital stay was marked by confabulation in addition to altered mental status, nystagmus, and an undetectable thiamine level, all symptoms were present before thiamine treatment had been started. Two patients did receive glucose before thiamine was given, but both had resolution of their symptoms at discharge. There were no reported adverse effects seen in any of the patients attributed to thiamine.

When comparing the duration of treatment with high-dose thiamine, patients whose symptoms resolved were treated for a median of three days versus two days in those patients with persistent symptoms. Interestingly, patients whose symptoms persisted despite high-dose thiamine treatment started highdose therapy at a median time of $90 \mathrm{~h}$ (range=69-111 h) after symptom onset compared to a median time of $92 \mathrm{~h}$ (range=46-186 h) until start of therapy from symptom onset in patients whose symptoms eventually improved.

\section{Discussion}

Pathophysiology of $W K S$. Thiamine plays a key role in several enzyme and metabolic pathways, including involvement with transketolase and glucose metabolism within the CNS $(9,10)$. Prolonged thiamine deficiency depletes the body stores within 2-3 weeks (11). As thiamine blood levels fall, thiamine-dependent enzyme systems involved in prevention of cellular damage become impaired and metabolic demands increase, which can result in selective brain lesions correlated with Wernicke's encephalopathy and Korsakoff's syndrome. Consequently, thiamine deficiency and development of WKS is particularly prevalent in malnourished patients oftentimes due to the shift in diet away from vitamin-rich foods and, as in the case of chronic alcoholism, a shift towards the carbohydrate-heavy consumption of alcohol.

Differential diagnosis. As stated, the classical triad symptoms of ocular signs, ataxia, and altered mental status is neither ubiquitous nor unique to WKS, but rather more diagnostically indicative of WKS when used in conjunction with the clinical picture. MRI signal characteristics (reversible cytotoxic edema typified by symmetric alterations in the thalami, mammillary bodies, tectal plate, and periacqueductal area) are still not definitive for a WKS diagnosis $(2,11,12)$. The differential diagnosis may include other encephalopathies, paramedian thalamic infarction, primary cerebral lymphoma, multiple sclerosis, Creutzfeldt-Jakob disease, and Leigh's disease, among others $(11,13,14)$. In 1986, the retrospective analysis of 131 WKS subjects by Harper et al. demonstrated the relatively poor ability to correctly diagnose WKS, as $80 \%$ of the patients who had confirmed WKS were diagnosed for the first time posthumously on necropsy (3). Because of its relatively non-specific and oftentimes poorly recognized clinical presentation of WKS, it is important to recognize the "at risk" patient population for developing WKS and to begin empiric treatment appropriately when the disease is suspected.

Acute treatment. It is established that thiamine replacement is the primary treatment for WKS in order to reverse mental status changes and prevent further disease progression. Parenteral thiamine is used in the acute treatment of Wernicke's since intestinal absorption of thiamine may be impaired, as in the case of alcoholics (1). A 1998 study demonstrated decreased expression of thiamine transporter1 , which is involved in thiamine uptake in the gut and inhibition of carrier-mediated thiamine uptake in murine models fed alcohol chronically, which supports the idea that 
Table I. Demographic and clinical characteristics of patients with Wernicke's encephalopathy.

\begin{tabular}{|c|c|c|c|c|c|c|c|c|}
\hline Case & $\begin{array}{l}\text { Age } \\
(\mathrm{yrs})\end{array}$ & Gender & Race & $\begin{array}{l}\text { Thiamine } \\
\text { level } \\
(\mathrm{nmol} / \mathrm{l})\end{array}$ & $\begin{array}{l}\text { Thiamine dosing } \\
\text { regimen }\end{array}$ & $\begin{array}{l}\text { Duration of } \\
\text { high-dose } \\
\text { therapy (days) }\end{array}$ & $\begin{array}{l}\text { Time to high-dose } \\
\text { thiamine } \\
\text { treatment (hours) }\end{array}$ & $\begin{array}{l}\text { Resolution of } \\
\text { symptoms }\end{array}$ \\
\hline 1 & 43 & $\mathrm{~F}$ & AA & $<7$ & 100 mg PO qDay, 500 mg IV qDay & 3 & 46.2 & Yes \\
\hline 2 & 44 & & AA & 22 & $\begin{array}{c}100 \mathrm{mg} \text { PO, } 500 \mathrm{mg} \text { IV q8h, } 500 \mathrm{mg} \text { IV qDay, } \\
100 \mathrm{mg} \text { PO qDay }\end{array}$ & 7 & 61.9 & Yes \\
\hline 3 & 64 & & $\mathrm{~W}$ & $94 *$ & $100 \mathrm{mg}$ PO, $500 \mathrm{mg}$ IV q8h, $500 \mathrm{mg}$ IV qDay & 7 & 84.4 & No \\
\hline 4 & 80 & & W & None & $100 \mathrm{mg}$ PO, $500 \mathrm{mg}$ IV BID, $500 \mathrm{mg}$ IV qDay & 8 & 117.4 & Yes \\
\hline 5 & 57 & & AA & None & $500 \mathrm{mg}$ IV once, $100 \mathrm{mg}$ PO qDay & 1 & 117 & Yes \\
\hline 6 & 48 & & AA & None & $100 \mathrm{mg}$ PO, $500 \mathrm{mg}$ IV q8h & 2 & 47.4 & Yes \\
\hline 7 & 63 & $\mathrm{~F}$ & AA & $<7$ & $\begin{array}{l}500 \text { mg IV q8h, } 200 \text { mg IV qDay, } \\
100 \text { mg PO qDay }\end{array}$ & 3 & 111.3 & No \\
\hline 8 & 67 & $\mathrm{~F}$ & $\mathrm{~W}$ & $<7$ & $500 \mathrm{mg}$ IV q8h, $100 \mathrm{mg}$ IV qDay & 2 & 186.4 & Yes \\
\hline 9 & 57 & $\mathrm{~F}$ & AA & $<7$ & $500 \mathrm{mg}$ IV q8h, $100 \mathrm{mg}$ PO qDay & 7 & 69 & No \\
\hline 10 & 69 & $\mathrm{~F}$ & AA & $<7$ & $500 \mathrm{mg}$ IV TID, $250 \mathrm{mg}$ IV qDay & 3 & 92 & Yes \\
\hline 11 & 58 & $\mathrm{~F}$ & AA & $507 *$ & $500 \mathrm{mg}$ IV TID, $500 \mathrm{mg}$ IV qDay & 3 & 96 & No \\
\hline
\end{tabular}

*Thiamine levels obtained after thiamine administered. ${ }^{\dagger}$ Defined as time between either admission or start of symptoms and high-dose thiamine therapy initiation. AMS, Altered mental status; IV, intravenous; PO, orally; q8h, every 8 hours; qDay, every day; BID, twice a day; TID, three times a day.

alcohol absorption is affected by alcohol intake. Furthermore, the same study showed similar uptake inhibition of thiamine using in vitro experiments of human intestinal epithelial cells chronically treated with ethanol (15). However, strong evidence supporting a defined dose and duration for thiamine in the treatment of Wernicke's encephalopathy is lacking.

Earlier studies on the acute treatment of WKS report an initial dose of 50-100 $\mathrm{mg}$ of intravenous thiamine as well as replacement of magnesium, since deficiency can result in impaired response to thiamine repletion $(1,7)$. However, later case studies showed that 100-250 mg doses of parenteral thiamine did not prevent death and that Korsakoff's psychosis developed in 56-84\% of patients later on follow-up $(5,16,17)$. A current dosing recommendation comes from a 1998 report by Cook et al. which had examined the current literature at the time on WKS, including fourteen studies on thiamine and B-complex vitamin use in Wernicke's encephalopathy. An intravenous dose of $500 \mathrm{mg}$ three times a day for two consecutive days was recommended, with a further five days of $500 \mathrm{mg}$ of intravenous or intramuscular thiamine given if a response in therapy was achieved (9). Furthermore, the British National Formulary and Royal College of Physicians in London recommend a similar regimen in accordance with guidelines by the British Association for Psychopharmacology, the European Federation of Neurological Societies (EFNS) and the National Institute for Healthcare and Excellence (NICE) (18-20). This dosing regimen calls as well, for $500 \mathrm{mg}$ intravenous thiamine three times daily for 2 to 3 days with a further $250 \mathrm{mg}$ intravenous thiamine for the next 2-3 days. The intravenous regimen is then followed by oral thiamine supplementation indefinitely (7).

This case series demonstrated that even though the majority of the subjects were given intravenous thiamine at the recommended dosages initially, resolution of symptoms was experienced in eight out of eleven patients with one patient likely experiencing Korsakoff's psychosis. Only one patient had suspicion of Wernicke's encephalopathy due to findings on MRI with the remainder of the subjects being treated empirically and based on clinical findings. Given the difficulties in the diagnosis of WKS coupled with unknown documentation of the time of onset of symptoms, it is challenging to determine whether treatment with thiamine was given in a sufficiently timely manner. Many patients were admitted with ongoing altered mental status and a history of other clinical signs, making ascertaining the exact timing of symptom onset difficult. Regardless, the time from suspected symptom onset to administration of high-dose thiamine did not appear markedly different between patients with symptom resolution and those without. Moreover, the duration of the high-dose thiamine treatment was longer in patients with improvement of symptoms, though the significance of this and transition to oral thiamine cannot be explored without follow-up in a larger patient population. Nevertheless, reversal and improvement of symptoms was observed in more than $70 \%$ of the subjects in this case series when treated with high-dose thiamine, suggesting that it is a viable treatment option in patients with a presumptive diagnosis of WKS. 


\section{Conclusion}

Due to high morbidity and mortality of WKS and limited adverse effects associated with parenteral thiamine, clinicians should start empiric therapy as soon as signs and symptoms are recognized. For the treatment of acute Wernicke's encephalopathy, our case series demonstrates that IV thiamine appears efficacious and safe for use, even in higher doses relative to traditional delirium tremens prophylactic dosing (100 mg/day thiamine). Within our institution, treatment doses and duration of thiamine used in the subjects were similar, but not uniform. More research is warranted to investigate the dose and duration of thiamine treatment as well as adjunct treatments that optimize therapeutic outcomes in this potentially life-threatening disease state.

\section{Conflicts of Interest}

The Authors have no conflicts of interest to disclose.

\section{References}

1 Zubaran C, Fernandes JG and Rodnight R: Wernicke-Korsakoff syndrome. Postgrad Med J 73: 27-31, 1997.

2 Zuccoli G, Gallucci M, Capellades J, Regnicolo L, Tumiati B, Giadas TC, Bottari W, Mandrioli J and Bertolini M: Wernicke encephalopathy: MR findings at clinical presentation in twentysix alcoholic and nonalcoholic patients. AJNR Am J Neuroradiol 28: 1328-1331, 2007.

3 Harper CG, Giles M and Finlay-Jones R: Clinical signs in the Wernicke-Korsakoff complex: a retrospective analysis of 131 cases diagnosed at necropsy. J Neurol Neurosurg Psychiatry 49: 341-345, 1986.

4 Zuccoli G, Siddiqui N, Bailey A and Bartoletti SC: Neuroimaging findings in pediatric Wernicke encephalopathy: a review. Neuroradiology 52: 523-529, 2010.

5 Victor M, Adams RD and Collins GH: The Wernicke-Korsakoff syndrome. A clinical and pathological study of 245 patients, 82 with post-mortem examinations. Contemp Neurol Ser 7: 1-206, 1971.

6 Victor M AR and Collins GH: The Wernicke-Korsakoff syndrome and related neurological disorders due to alcoholism and malnutrition. 2nd ed. Philadelphia, PA: FA Davis.

7 Thomson AD, Guerrini I and Marshall EJ: The evolution and treatment of Korsakoff's syndrome: out of sight, out of mind? Neuropsychol Rev 22: 81-92, 2012.

8 Institute of Medicine (U.S.). Standing Committee on the Scientific Evaluation of Dietary Reference Intakes., Institute of Medicine (U.S.). Panel on Folate Other B Vitamins and Choline., Institute of Medicine (U.S.). Subcommittee on Upper Reference Levels of Nutrients. Dietary reference intakes for thiamin, riboflavin, niacin, vitamin B6, folate, vitamin B12, pantothenic acid, biotin, and choline. Washington, D.C.: National Academy Press; 1998.
9 Cook CC, Hallwood PM and Thomson AD: B Vitamin deficiency and neuropsychiatric syndromes in alcohol misuse. Alcohol Alcohol 33: 317-336, 1998.

10 Wijnia JW and Oudman E: Biomarkers of delirium as a clue to diagnosis and pathogenesis of Wernicke-Korsakoff syndrome. Eur J Neurol 20: 1531-1538, 2013.

11 Sechi G and Serra A: Wernicke's encephalopathy: new clinical settings and recent advances in diagnosis and management. Lancet Neurol 6: 442-455, 2007.

12 Donnal JF, Heinz ER and Burger PC: MR of reversible thalamic lesions in Wernicke syndrome. AJNR Am J Neuroradiol 11: 893894, 1990.

13 Brechtelsbauer DL, Urbach H, Sommer T, Blumcke I, Woitas R and Solymosi L: Cytomegalovirus encephalitis and primary cerebral lymphoma mimicking Wernicke's encephalopathy. Neuroradiology 39: 19-22, 1997.

14 Weidauer S, Nichtweiss M, Lanfermann H and Zanella FE: Wernicke encephalopathy: MR findings and clinical presentation. Eur Radiol 13: 1001-1009, 2003.

15 Subramanya SB, Subramanian VS and Said HM: Chronic alcohol consumption and intestinal thiamin absorption: effects on physiological and molecular parameters of the uptake process. Am J Physiol Gastrointest Liver Physiol 299: G23-31, 2010.

16 Wood B, Currie J and Breen K: Wernicke's encephalopathy in a metropolitan hospital. A prospective study of incidence, characteristics and outcome. Med JAust 144: 12-16, 1986.

17 Thomson AD, Cook CC, Guerrini I, Sheedy D, Harper C and Marshall EJ: Wernicke's encephalopathy: 'Plus ca change, plus c'est la meme chose'. Alcohol Alcohol 43: 180-186, 2008.

18 Thomson AD, Cook CC, Touquet R, Henry JA and Royal College of Physicians L: The Royal College of Physicians report on alcohol: guidelines for managing Wernicke's encephalopathy in the accident and Emergency Department. Alcohol Alcohol 37: 513-521, 2002.

19 Galvin R, Brathen G, Ivashynka A, Hillbom M, Tanasescu R, Leone MA and EFNS: EFNS guidelines for diagnosis, therapy and prevention of Wernicke encephalopathy. Eur J Neurol 17: 1408-1418, 2010.

20 NICE Clinical Guidelines, No. 115. Alcohol-Use Disorders: Diagnosis, Assessment and Management of Harmful Drinking and Alcohol Dependence. National Collaborating Centre for Mental Health. Leicester (U.K.), 2011.
Received October 19, 2016

Revised November 15, 2016

Accepted December 13, 2016 